\title{
Prevalence of Disease and Antibiotic Sensitivity Profile of Hospital Associated Pathogens: A study on a local diagnostic Centre in Dhaka city
}

\author{
Sharmin Ahmed, Riajul Islam and Sunjukta Ahsan* \\ Department of Microbiology, University of Dhaka, Dhaka-1000, Bangladesh,
}

\begin{abstract}
Different types of diseases inflict the inhabitants of Dhaka city from time to time. The focus of this study was to investigate disease prevalence, etiology and antibiotic sensitivity profiles reported at a local diagnostic service in Dhaka, Bangladesh. Pathogens were transported from a renowned local diagnostic centre in Dhaka city in Trypticase Soy broth (TSB) to the laboratory. Antibiogram was performed by Kirby Bauer disc diffusion assay. A total of 110 samples $(20 \%$ blood $(n=22), 60.9 \%$ urine $(n=67), 13.64 \%$ pus $(n=15), 3.64 \%$ sputum $(n=4)$ and $1.82 \%$ wound swab samples $(n=2)$ were collected for further investigation. Of the patients, $30 \%$ were male and $70 \%$ were female. In case of blood samples from diarrheal patients, Salmonella enterica serovar Typhi (72.7\%) and $S$. enterica serovars Paratyphi (27.2\%) were predominant. Both serovars were sensitive to Cefixime, Ceftriaxone and Gentamicin and resistant to Nalidixic acid. $E$. coli $(\mathbf{9 1 \% )}$ was the predominant pathogen in Urinary Tract Infection (UTI) patients followed by Klebsiella spp. (8.9\%). E. coli exhibited resistance to Cephalosporins and Ciprofloxacin whereas Klebsiella spp. were, however, sensitive to these antibiotics. Both species showed resistance against Amoxicillin and sensitivity to Imipenem, Merpenem and Colistin. In pus samples, S. aureus $(46.7 \%)$ prevailed followed by Klebsiella spp. (26.7\%) and $E$. coli $(26.7 \%)$ spp. All $S$. aureus isolates were sensitive to Fusidic acid, Vancomycin, Linezolid and Piperacillin and $\mathbf{5 0 \%}$ were sensitive to Cephalosporins and Ciprofloxacin and resistant to Amoxycillin and Azithromycin. E. coli and Klebsiella spp. from pus samples could be inhibited by Carbapenem. As with UTI isolates, E. coli from pus were resistant to Cephalosporins, Ciprofloxacin and Amoxicillin whereas $66 \%$ Klebsiella spp. were sensitive to the first two. A total of $66 \%$ of $E$. coli and Klebsiella spp. showed sensitivity to Gentamicin and Amikacin. It was observed that there was a similarity in the antibiotic sensitivity pattern of the same bacterial spp. isolated from different disease cases. As a general rule, it was observed that Imipenem is a good treatment option for treating $E$. coli and Klebsiella spp., Cephalosporins for Salmonella while a number of treatment options existed for S. aureus infections.
\end{abstract}

Key words: pathogens, antibiogram, etiologic agent

\section{Introduction}

Dhaka is the capital city of Bangladesh. It is one of the biggest and densely populated cities in the world with a population of 20.2 million people in the Greater Dhaka Area ${ }^{1}$. While urban life offers many amenities, urban areas can also ponder health hazards to its inhabitants. Urban populations in both low- and high- income countries disclose some of the world's most noticeable health inequalities.

Many urban dwellers live in congested slums and places that deficient from proper sanitation and healthy housing. Unplanned urban housing, transport, and food systems, along with social and lifestyle factors, are drivers in the epidemic of noncommunicable and communicable diseases, which are linked to risks and hazards such as air pollution, poor diet, physical idleness, traffic injury and domestic injury ${ }^{2}$. So, it is necessary to know about the city dwellers health conditions and the causative agents involves in their diseases. This can be done by epidemiological study on diseases prevalence to investigate the predominant pathogen and their pattern of antibiotic sensitivity. The prevalence of a disease is one of the most essential measures in epidemiology. The term prevalence defines the burden of disease in a population in a particular location at a particular time. It is important to count the number of people affected with a disease so that the appropriate planning and health care needs can be provided to affected people as early as possible ${ }^{3}$.

Inhabitants of Dhaka city are exposes to various etiologic agents from time to time. Seasonal variation also influences the emergence of diseases because the maintenance of virulence by any pathogen outside the host is dependent on a number of factors, viz. temperature, moisture, dehydration, and UV light. Of these factors, temperature has the greatest effect, since the rate of most chemical and physical processes are depend on it ${ }^{4}$. One of the major challenges in disease managements is the emergence of drug-resistant bacteria, posing an important impression on the effectiveness of chemotherapy. Through the past few years, the antibacterial activity of a number of medications has reduced with the concomitant onset of the drug-resistant pathogenic bacteria ${ }^{5}$. This has become the most severe public health concerns worldwide, leading to mortalities from meek microbial infections followed by treatment-mediated difficulties from inactive drugs. 
The increase in antibiotic resistance is now one of the top three greatest threats to global health in our time ${ }^{6}$. In 2014, the World Health Organization (WHO) published a report on the global surveillance of antimicrobial resistance ${ }^{7}$. The worldwide increases in single drug-resistant bacteria, multidrug-resistant (MDR) bacteria, and extensively drug-resistant (XDR) bacteria are indeed well-known. For example Staphylococcus aureus has been recognized as a main pathogen of hospital acquired infections. MRSA (methicillin-resistant Staphylococcus aureus) strains have become endemic in hospitals worldwide. In previous time Methicillin a beta lactamase inhibitor drug was the treatment of choice against $S$. aureus but now it's no longer effective In addition, it is now incipient community pathogen in many geographical regions ${ }^{8}$.

Extended spectrum beta-lactamase (ESBL) producing organisms are big concern for health care providers due to no effect of microbes on the cephamycins and carbapenems ${ }^{9}$. They are increasing rapidly and becoming a major problem in the area of infectious diseases. Problems associated with ESBL producing isolates are difficult to be detected or treated, thereby causing increased mortality of patients ${ }^{10}$. Since a national investigation system is lacking in Bangladesh on antibiotic resistance and the pattern of resistance among etiologic agents of various diseases, there is a great need for long-term studies on the proportions and trends in antibiotic resistance.

\section{Material and methods}

\section{Study population}

A cross sectional retrospective study was done among patients to investigate disease prevalence, etiology and antibiotic sensitivity profiles reported at a local diagnostic service named Medinova diagnostic center in Dhaka city for August 2015 to September 2015. During this time period a total of 110 samples were collected aseptically from patients ( 73 women, 37 men) by hospital personnel. The patients were from different age groups.

\section{Sample types}

Four types of samples were considered: urine, blood, pus, wound swab and sputum. Samples were collected by professionals and collected bacteria were provided for the present study.

\section{Culture maintenance and antibiotic sensitivity test}

From collected samples bacteria were isolated and presumptive identification was done by culture and biochemical method and isolated pathogenic microbes were carried in Trypticase Soy broth (TSB) during transport to the laboratory. Isolates were preserved in glycerol broth $(700 \mu \mathrm{l}$ of $95 \%$ glycerol and $300 \mu \mathrm{l}$ bacterial log phase culture) for antimicrobial susceptibility testing by disk diffusion method. The antibiotic sensitivity pattern of each isolates were interpreted according to CLSI guideline. Reference strains of $E$. coli ATCC 25922 was used for quality control for antimicrobial susceptibility tests.

\section{Results}

A total of 110 samples were collected from a local renowned diagnostic center. Female patients were prevalent than male. Of them, $30 \%$ were male and $70 \%$ were female. Figure 1 (a) and (b) show the prevalence of sex and sample types and their distribution pattern in study population.

Blood isolates: Blood samples were collected from diarrheal patients. Among blood isolates, Salmonella enterica serovar Typhi (72.7\%) and S. enterica serovars Paratyphi (27.2\%) were predominant. Antibiotic sensitivity test was done against multiple antibiotics such as: Ampicillin (10 $\mu \mathrm{g})$, Azithromycin $(15 \mu \mathrm{g})$, Cefixime $(5 \mu \mathrm{g})$, Ceftriaxone $(30 \mu \mathrm{g})$, Chloramphenicol $(30 \mu \mathrm{g})$, Gentamycin $(10 \mu \mathrm{g})$, Nalidixic acid (30 $\mu \mathrm{g})$ (Figure 2).

It was observed that both serovars were sensitive to Cefixime, Ceftriaxone and Gentamicin but resistant to Nalidixic acid. Figure 2 presents the antibiotic sensitivity profile of Salmonella enterica serovars Typhi and S. enterica serovars Paratyphi isolated from blood samples of diarrheal patients.

Pus isolates: From pus samples various isolates were found and among them $S$. aureus (46.7\%) ruled in number followed by Klebsiella spp. (26.7\%) and E. coli (26.7\%) spp. Antibiotics which were tested against these isolates were Amoxyclave (30 $\mu \mathrm{g})$, Amoxicillin $(10 \mu \mathrm{g})$, Azithromycin $(15 \mu \mathrm{g})$, Cefixime $(5 \mu \mathrm{g})$, Cefotaxime $(5 \mu \mathrm{g})$, Ceftazidime $(30 \mu \mathrm{g})$, Ceftriaxone $(30 \mu \mathrm{g})$, Cephalexine $(30 \mu \mathrm{g})$, Ciprofloxacin $(10 \mu \mathrm{g})$, Cloxacillin $(5 \mu \mathrm{g})$, Fusidic acid $(10 \mu \mathrm{g})$, Gentamycin $(10 \mu \mathrm{g})$, Cotrimoxazole $(25 \mu \mathrm{g})$,Vancomycin $(30 \mu \mathrm{g})$, Linezolid $(30 \mu \mathrm{g})$, Piperacillin (36 $\mu \mathrm{g})$ and Colistin $(50 \mu \mathrm{g})$ (Figure 3).
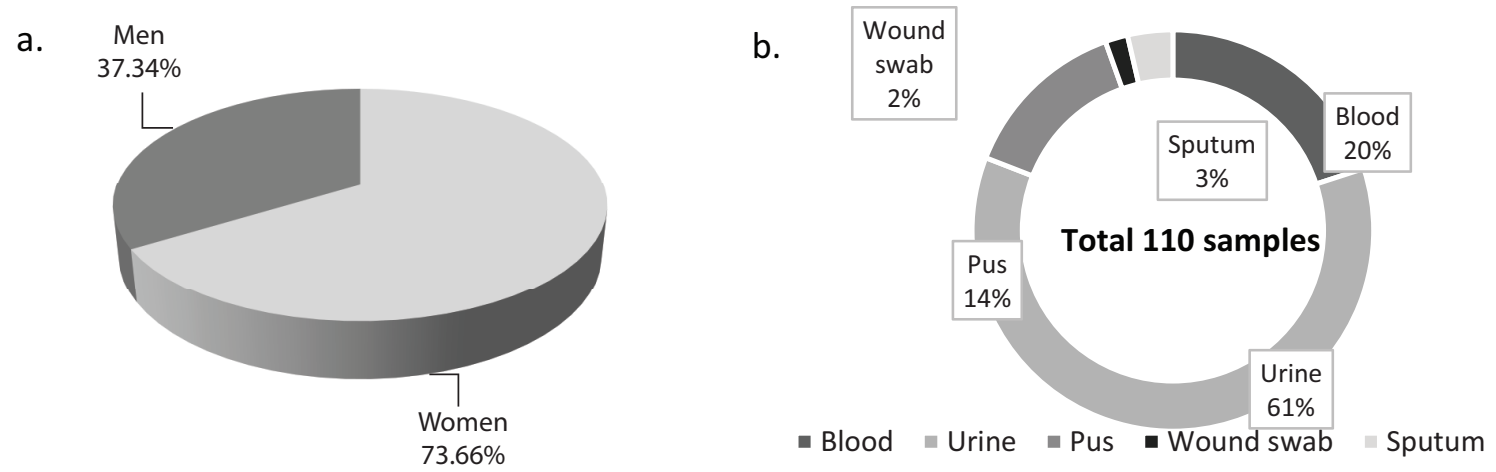

Figure 1. (a) The proportion of male and female patients; (b) The proportion of samples collected from different sources. 


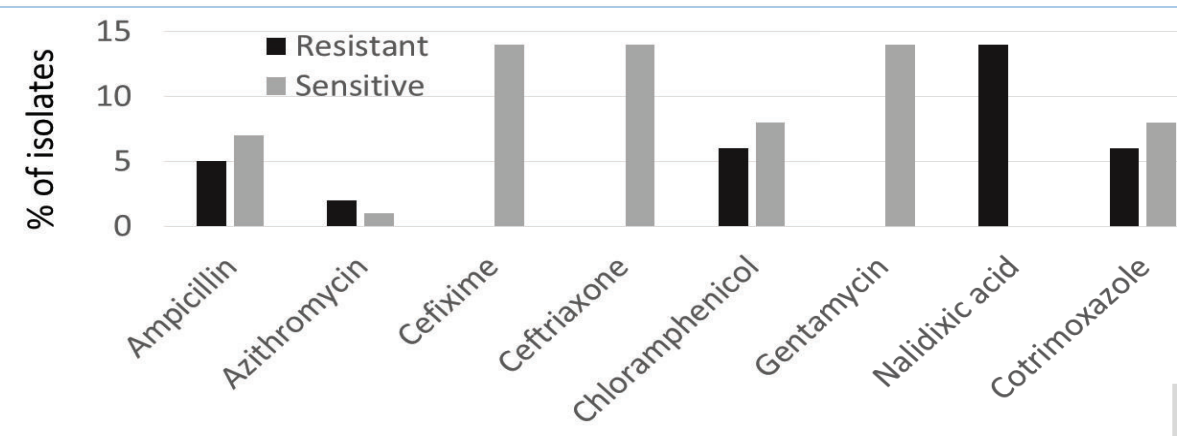

(a)

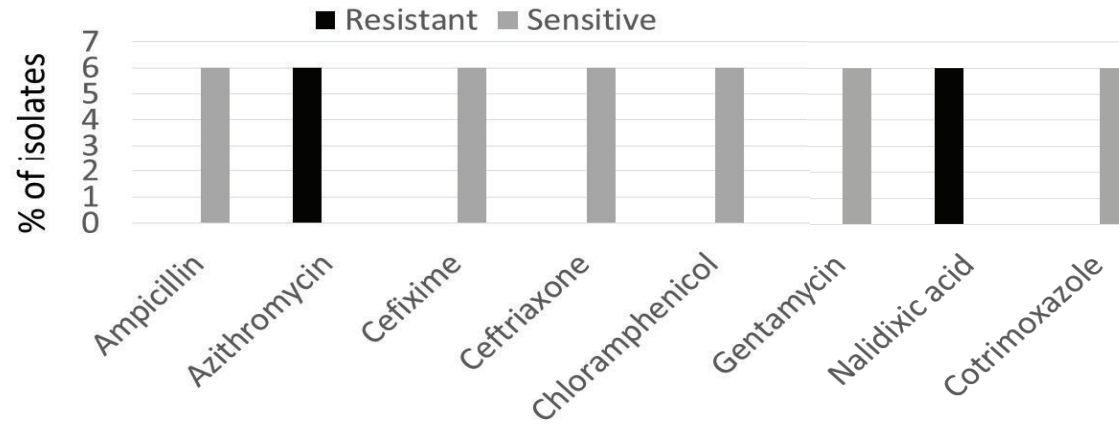

Figure 2. Antibiotic sensitivity pattern of blood isolate (a) S. enterica serovar Typhi, (b) S. enterica serovar Paratyphi
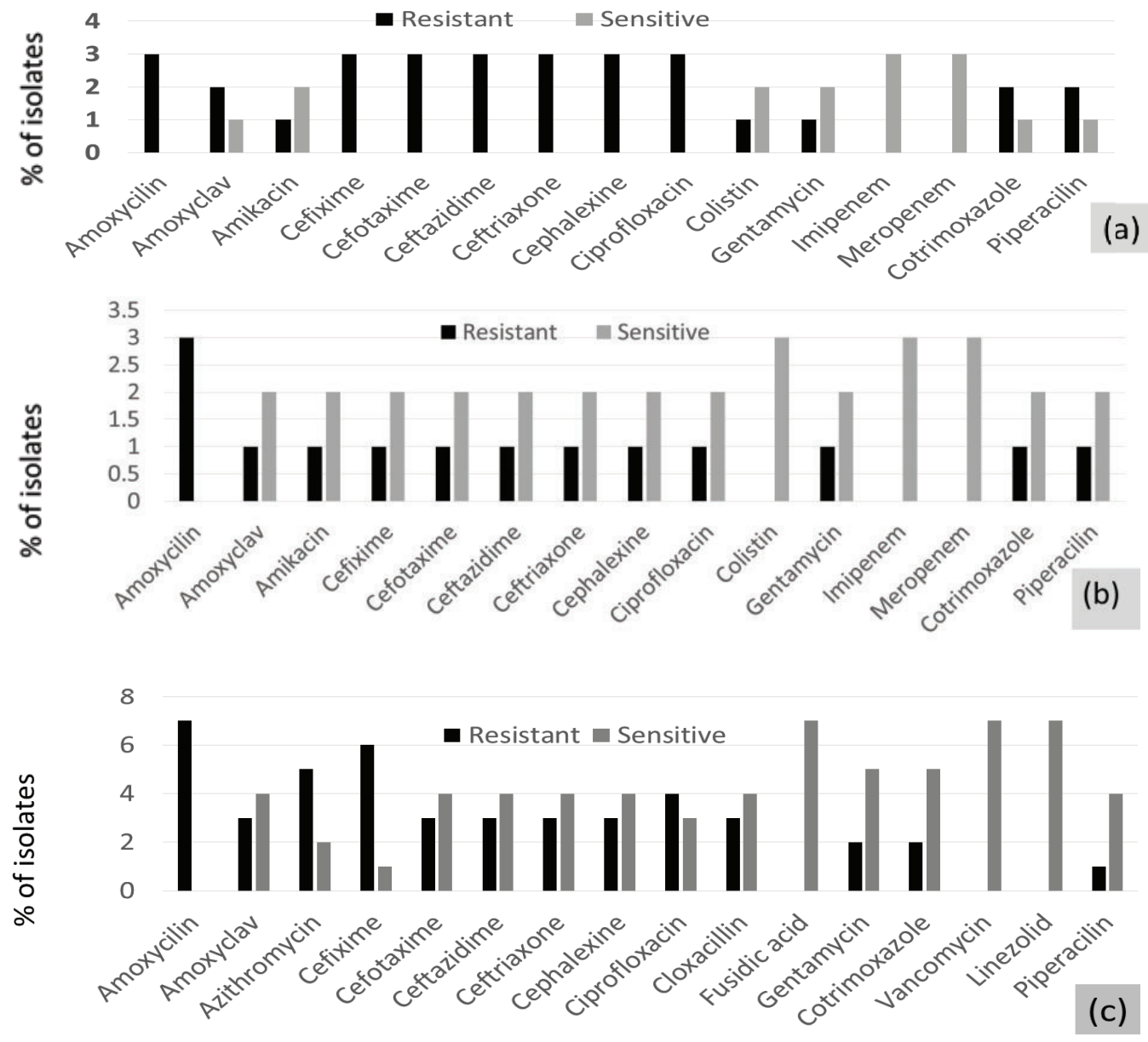

Figure 3. Antibiotic sensitivity pattern of Pus isolates (a) E. coli, (b) Klebsiella spp. (c) S. aureus 
(a)
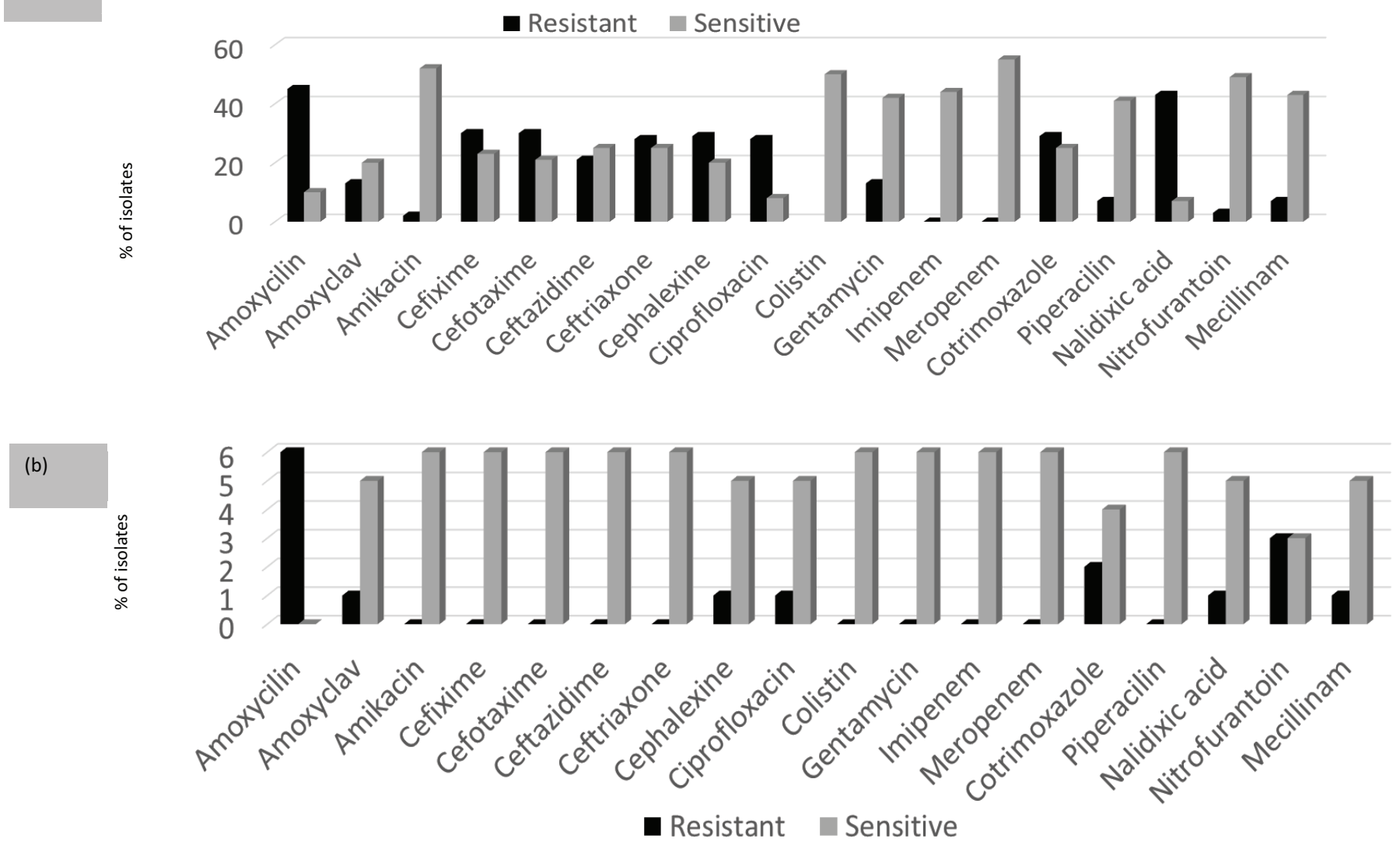

Figure 4. Antıbiotlc sensitıvity pattern of Urine isolates (a) t. coll (b) Klebslella spp.

Figure 3 which shows antibiotic sensitivity pattern of pus isolates witnessed that all $S$. aureus were sensitive to Fusidic acid, Vancomycin, Linezolid and Piperacillin and 50\% were sensitive to Cephalosporins and Ciprofloxacin and resistant to Amoxycillin and Azithromycin. But E. coli and Klebsiella spp. from pus samples were inhibited by Carbapenem and Colistin. E. coli exhibited greater resistance to various antibiotics than Klebsiella spp.

Urine isolates: As with pus and blood, urine samples were taken from UTI patients and from which E. coli (91\%) and Klebsiella spp. ( $9 \%$ ) were found. As with pus isolates, E. coli from UTI were resistant to Cephalosporins, Ciprofloxacin and Amoxicillin whereas $66 \%$ Klebsiella spp. were sensitive to the first two. The result of antibiotic sensitivity profile of UTI isolates were given in figure 4.

A total of $66 \%$ of E. coli and Klebsiella spp. showed sensitivity to Gentamicin, Amikacin, Carbapenems and it was observed that irrespective of its source, the bacteria from pus or UTI patient same bacteria from different samples for different infection they showed similar sensitivity pattern against various antibiotics. $2^{\text {nd }}$ and $3^{\text {rd }}$ generation cephalosporin drugs were no longer active against them whereas carbapenem showed maximum activity against all regardless of infection and sample types.

\section{Discussion}

The study was done in a one month study period to observe the diseases prevalence and antibiotic sensitivity profile of their causative agents. In this study period samples were taken from a well-known diagnostic center and it was showed the distribution pattern of diseases and pathogenic bacterial species in city population. From 110 patients including male and female four types of samples were taken from blood, urine, pus, sputum.

Patients affected by diarrheal infection, from them blood samples were collected and predominant isolates were found Salmonella enterica serovar Typhi and S. enterica serovars Paratyphi. The study period was august 2015 to September 2015 and it was summer time in Bangladesh. Among patients women were more prevalent as they were exposed to outside unhygienic food, unconscious to health issues than men.

Antibiotic resistance is a major problem in Salmonella enterica serovar Typhi, the causative agent of typhoid. Multidrugresistant (MDR) isolates are prevalent in parts of Asia and Africa. Reduced susceptibility to fluoroquinolones is also widespread, and sporadic cases of resistance to third-generation cephalosporins or azithromycin have also been reported ${ }^{11}$.This finding is also relevant with our study result. In our study the antibiotic sensitivity pattern presented that $S$. enterica serovar Typhi was more resistant than S. enterica serovar Paratyphi. But 
they showed similar pattern of sensitivity against same drug. For example the isolated $S$. enterica serovar Typhi and S. enterica serovar Paratyphi both were completely resistant to Nalidixic acid and Azithromycin. About $50 \%$ of S. enterica serovar Typhi showed resistance against Cotrimoxazole, Chloramphenicol, and Ampicillin. Whereas S. enterica serovar Paratyphi showed complete sensitivity against those drugs. Both types of bacteria showed $100 \%$ sensitivity against Cefixime, Ceftriaxone and Gentamycin.

Staphylococcus aureus is a human pathogen that reasons skin and soft tissue abscesses. Staphylococci in pus spread onto skin surfaces or pass in circulating lymph and blood, which cause formation of abscesses at new sites Abscess formation is not distinctive to staphylococcal infection and purulent discharge has been extensively considered a physiological sign of healing. But it was seen that some virulence factors of $S$. aureus is responsible for abscesses formation ${ }^{12}$. Among pus isolates in our study $S$. aureus, E.coli and Klebsiella sp. were predominant. E. coli expressed more resistance to multiple antibiotics than Klebsiella sp. From antibiotic sensitivity profile it was observed that Imipenem and Meropenem could be the treatment of choice for purulent pus infections when causative agents are E. coli and Klebsiella sp. On other hand Linezolid, Fusidic acid, Vancomycin presented $100 \%$ affectivity against $S$. aureus. the enormous use of antibiotics above the past 50 years has led to the creation of drug-resistant strains, designated MRSA for methicillinresistant $S$. aureus, so it is no longer in clinical use in the United States and in other countries ${ }^{13}$. Regardless of whether it was gram negative or gram positive bacteria, in our study it was observed that amoxicillin was no longer in the list of drug of choice for pus infection treatment.

Urinary tract infections (UTIs) are one of the most frequent infections worldwide. Escherichia coli is the most common UTI pathogen although patients' age and gender may impact prevalence of causative agents. They increase accuracy in defining the causative pathogen and provide help to empiric treatment ${ }^{14}$.

Urine samples were taken from UTI patients of all age groups and affected patients were predominantly female. UTIs occur more in women than in men, at a ratio of 8:1 due to anatomical position of body ${ }^{15}$. In our study we were found E. coli and Klebsiella sp. from urine samples were E. coli played the major role as infectious agent. Other causative agents of UTI that are reported in other studies are Proteus mirabilis, Staphylococcus saprophyticus, and Staphylococcus epidermidis. Klebsiella and group B streptococcus infections in diabetic patients and Pseudomonas infections occurred in chronically-catheterized patients have already been reported ${ }^{16}$.

In case of urine samples, $80 \%$ of isolates in our study showed sensitivity against Piperacillin, Colistin, Amikacin, Gentamycin, Meropenem and Imipenem. As with pus isolate similar pattern of resistance against amoxicillin and cephalosporins were presented by urine isolates which was relevant with present reported studies.it has been reported that Ampicillin, amoxicillin, sulfonamides are no longer the drugs of choice for treatment of UTI because of the extensive occurrence of resistance in 15$20 \%$ of $E$. coli in areas of USA and other countries ${ }^{17,18}$.

Research on urinary tract infections (UTIs) in West Africa from 1990 to 2012 have presented moderate to high antimicrobial resistance to commonly prescribed antibiotics. Fluoroquinolones have been suggested in the management of UTIs, but recent reports demonstrated the emergence of resistance. Levofloxacin and ciprofloxacin still remain the commonest fluoroquinolones prescribed for UTIs in many settings ${ }^{19}$. But resistance is rapidly occurring against this drugs.

As third-generation oral cephalosporins, cefixime is active against microbes causing community-acquired UTIs, and is only hydrolyzed by extended spectrum ${ }^{2}$-lactamases and high levels of chromosomally mediated cephalosporinase ${ }^{20}$. But in our study it was seen that $E$. coli from UTI samples showed higher resistance to this drugs due to its excessive uses.

In conclusion our data suggest that there was a resemblance in antibiotic sensitivity profile of same bacterial species isolated from different types of samples from different patients affected with different diseases conditions. It was observed that Imipenem can be treatment option for treating E. coli and Klebsiella spp., whereas Cephalosporins can be a drug of choice for fluorequinolone resistant Salmonella and a number of treatment options existed for S. aureus infections such as Linezolid and Fusidic acid.

\section{References}

1. Choguill CL. 2012. New Communities for Urban Squatters: Lessons from the plan that failed in Dhaka, Bangladesh. Springer Science \& Business Media.

2. https://www.who.int/sustainable-development/cities/health-risks/en/

3. Ward MM. 2013. Estimating disease prevalence and incidence using administrative data: some assembly required. J Rheumatol. 40(8): 1241-3

4. Sinclair R, Boone SA, Greenberg D, Keim P and Gerba CP. 2008. Persistence of category A select agents in the environment. Appl Environ Microbiol. 74(3): 555-563.

5. Yaseen IH, Shareef AY and Daoud AS. 2013. High prevalence of multidrug-resistance MRSA and VRSA of different infections from AlJumhuory teaching hospital patients in Mosul. J Life Sci: 7:1255-9.

6. WEF, FEM. 2013. Global risks 2013. World Economic Forum.

7. World Health Organization. 2014. Antimicrobial resistance: global report on surveillance. World Health Organization.

8. Lowy FD. 1998. Staphylococcus aureus infections. New Eng J Med. 339(8): 520-532

9. Mukherjee M, BaSu S, Mukherjee SK and Majumder M. 2013. Multidrugresistance and extended spectrum beta-lactamase production in uropathogenic $E$. coli which were isolated from hospitalized patients in Kolkata, India. JCDR 7(3): 449.

10. Krishnakumar S, Rajan RA, Babu MM and Bai VD. 2012. Antimicrobial susceptibility pattern of extended spectrum of beta lactamase (ESBL) producing uropathogens from pregnant women. Indian J Med Healthcare. 1:188-92. 
11. Klemm, EJ, Shakoor, S, Page AJ, Qamar, FN, Judge K, Saeed DK and Shaheen G. 2018. Emergence of an extensively drug-resistant Salmonella enterica serovar Typhi clone harboring a promiscuous plasmid encoding resistance to fluoroquinolones and third-generation cephalosporins. MBio. 9(1): e00105-18.

12. Götz F, Bannerman T and Schleifer KH. 2006. The prokaryotes: a handbook on the biology of bacteria.

13. Brumfitt W and Hamilton-Miller J. 1989. Methicillin-resistant Staphylococcus aureus. New Eng J Med. 320(18): 1188-1196.

14. Magliano E, Grazioli V, Deflorio L, Leuci AI, Mattina R, Romano P and Cocuzza CE. 2012. Gender and age-dependent etiology of communityacquired urinary tract infections. Sci World J. 2012: 349597.

15. Rahn DD. 2008. Urinary tract infections: contemporary management. Urol Nurs. 28(5): 333-41.

16. Ronald A. 2002. The etiology of urinary tract infection: traditional and emerging pathogens. The Am J Med. 113(1): 14-19.
17. Gupta K, Hooton TM, Naber KG, Wullt B, Colgan R, Miller LG and Soper DE. 2011. International clinical practice guidelines for the treatment of acute uncomplicated cystitis and pyelonephritis in women: a 2010 update by the Infectious Diseases Society of America and the European Society for Microbiology and Infectious Diseases. Clin Infect Dis. 52(5): e103-e120

18. Kallen AJ, Welch HG and Sirovich BE. 2006. Current antibiotic therapy for isolated urinary tract infections in women. Arch Int Med. 166(6): 635639.

19. Afriyie DK, Adu LB, Dzradosi M, Amponsah SK, Ohene-Manu P and Manu-Ofei F. 2018. Comparative in vitro activity of ciprofloxacin and levofloxacin against isolated uropathogens in Ghana: a pilot study. Pan Afr Med J. 30: 194.

20. Garcia-Rodriguez JA and Bellido JM. 2000. Oral cephalosporins in uncomplicated urinary tract infections. Clin Microbiol Infect. 6: 73-75. 